\title{
Kernos
}

Revue internationale et pluridisciplinaire de religion grecque antique

4 | 1991

Varia

\section{« Mythe » et « rite » en Grèce : des catégories indigènes?}

\section{Claude Calame}

\section{(2) OpenEdition}

\section{Journals}

Édition électronique

URL : http://journals.openedition.org/kernos/299

DOI : $10.4000 /$ kernos.299

ISSN : 2034-7871

\section{Éditeur}

Centre international d'étude de la religion grecque antique

\section{Édition imprimée}

Date de publication : 1 janvier 1991

Pagination : 179-204

ISSN : 0776-3824

\section{Référence électronique}

Claude Calame, « « Mythe » et « rite » en Grèce : des catégories indigènes ? », Kernos [En ligne],

4 | 1991, mis en ligne le 11 mars 2011, consulté le 01 mai 2019. URL : http://journals.openedition.org/ kernos/299 ; DOl : 10.4000/kernos.299 
Kernos, 4 (1991), p. 179-204.

\section{«MYTHE» ET «RITE» EN GRÈCE : DES CATÉGORIES INDIGÈNES ?}

\section{De la traduction culturelle des taxinomies}

Chaque culture, on le sait, découpe à sa manière de vastes domaines de la réalité; rien de plus relatif à une communauté culturelle, donc, qu'une taxinomie. Chaque civilisation possède en propre ses systèmes de classification et, en raison même de sa relativité culturelle, toute nomenclature résiste à la traduction. Quoi de commun entre le système homérique de désignation des couleurs et la classification obéissant à la gradation des couleurs spectracles? Pas d'isomorphisme non plus entre la taxinomie botanique élaborée par Aristote et la nomenclature de Linné ${ }^{1}$.

Ce constant décalage, on en subit les incertitudes à deux égards, en particulier en anthropologie de l'Antiquité. Il s'agit en effet de traduire en un langage propre, dépendant d'un découpage culturel singulier, un ensemble de concepts dont articulation et désignation sont différentes. Aux difficultés inhérentes à toute transposition d'une langue à l'autre s'ajoutent donc les contradictions relatives à la théorie sémantique du Wortfeld telle que l'a développée entre les deux guerres J. Trier. Que va-t-on privilégier dans les ensembles taxinomiques, les lignes de démarcation tracées par les signifiants ou les limites dessinées par leurs signifiés ? le champ lexical ou le champ sémantique à proprement parler ? En admettant, comme le fait Trier, que par sa langue chaque culture opère à chaque moment de son histoire un découpage original dans le continu informe de la réalité, se fera-t-on l'adepte d'une théorie de la Weltanschauung annonciatrice de la fameuse thèse de Sapir et Whorf ? Ce serait faire fi de ces qualités distinctives différenciant les entités du monde naturel, indépendamment d'une langue envisagée comme moteur unique d'une vision significative ${ }^{2}$.

1 Les problèmes posés par la traduction des taxinomies d'une culture à l'autre sont évoqués par exemple par G. CaRdona, La foresta di piume. Manuale di etnoscienza, Roma - Bari, 1985, p. 35 sq.

2 J. Trier a exposé sa méthode notamment dans Das sprachliche Feld. Eine Auseinandersetzung, in Neue Jb. für Wiss. u. Jugendbildung, 10 (1934), p. 428449 , avec les remarques complémentaires de H. GECKELER, Strukturelle 
Le problème de la traduction lexicale et sémantique des catégories réelles ou conceptuelles d'une autre culture se complique quand ces classes ont été choisies pour fonder celles de la culture propre! C'est précisément le cas dans le domaine de l'anthropologie culturelle et sociale qui désigne deux de ses concepts fondateurs - le mythe et le rite par des termes empruntés à la culture de l'Antiquité. Puisant aux sources de l'historicisme romantique, elle a voulu ancrer dans la culture gréco-romaine quelques-uns de ses concepts opératoires les plus importants. Cette utilisation de signifiants aux assonances hellènes ou latines entretient donc l'illusion, par projection, que les catégories ainsi désignées y trouvent leur origine; ou davantage : qu'elles y correspondent à une réalité institutionnelle. Non content de renvoyer à une catégorie de récits dont les Grecs auraient été les fondateurs, le $\mu \hat{v} \theta \mathrm{o}$ est ainsi devenu l'emblème d'un type de pensée qui, prérationnel, s'opposerait aux lumières de la raison subsumées par un autre terme grec, celui de $\lambda$ ó $\gamma o \varsigma$. Abusivement attribué aux Hellènes, ce passage du

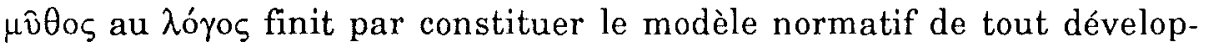
pement culturel. Il en va un peu différemment du rite pour qui on a privilégié, dans le champ lexical vaste de la désignation latine de la pratique cultuelle, un terme relativement rare, celui de ritus. Ainsi désignée, cette catégorie a été rapatriée dans le domaine hellène par la théorie anthropologique de J. Harrison; dans une perspective génétique et grâce à des termes qu'on verra empruntés au domaine restreint de la description des cultes à mystère, le rituel devient le $\delta \rho \omega ́ \mu \varepsilon v o v, ~ l ' « a g i »$ d'un mythe réduit comme $\lambda \varepsilon \gamma o$ ó $\varepsilon$ vov à l'état de son "dit", de son commentaire. Ce rapatriement induit du même coup une théorie très restreinte du mythe qui ne peut être compris que comme oitiıv - encore un terme grec -, que comme compte rendu étiologique de la pratique rituelle ${ }^{3}$ !

Semantik und Wortfeldtheorie, München, 1971, p. 115 sq. et p. 192 sq. Les progrès de la sémantique, notamment par l'analyse componentielle, ont permis de réduire une partie des difficultés rencontrées par TRIER : cf. P.R. LUTZEIER, Die semantische Struktur des Lexikons, in Ch. ScHWARZE und D. WuNDERLICH (edd.), Handbuch der Lexikologie, Königstein/Ts., 1985, p. 103-133. - Pour l'hypothèse SAPIR-WHORF, cf. en particulier B. L. WHORF, Linguistique et anthropologie. Les origines de la sémiologie, Paris, 1969 (éd. or. : Cambridge Mass., 1956), p. 69 sq., avec le commentaire de M. KILANI, Introduction à l'anthropologie, Lausanne, 1989, p. 144 sq. G. Mounin, Les problèmes théoriques de la traduction, Paris, 1963, p. 71 sq., pose dans une perspective ethnologique les problèmes de communicabilité entre cultures différentes.

3 Dans son analyse sémantique du terme, R. GANSCHINIETZ, art. Ritus, in RE, I A (1914), col. 924-934, montre bien d'une part le spectre très large recouvert par 
On aimerait donc se demander ici, dans la perspective de l'approche d'une culture différente et en l'espace limité d'un article, si les Grecs anciens ont conçu et s'ils ont disposé de notions correspondant plus ou moins à nos concepts de mythe et de rite. Sans avoir la prétention de recouvrir tout le développement historique des champs lexicaux et des champs sémantiques concernés, on se limitera à marquer quelques étapes dans les variations réciproques connues par les classes des Grecs et leurs signifiants. Prenant pour point de départ deux catégories centrales, en dépit de leur caractère flou, de l'anthropologie sociale contemporaine, ce sont les résultats d'un bref travail de traduction culturelle que l'on voudrait présenter ici : d'abord le mythe envisagé comme un éventuel genre narratif à caractère de fiction, puis le rite considéré en tant que pratique cultuelle et festive réglée ${ }^{4}$.

\section{Récits “mythiques»}

Supposée coïncider avec l'emploi du terme $\mu \hat{v} \theta_{\circ}$, la notion grecque de mythe a été soumise à de nombreuses études critiques. L'une des dernières en date a révélé les valeurs ethnocentriques incarnées dans un concept occidental de mythologie dont on a tenté d'attribuer la paternité aux Anciens ${ }^{5}$. Mais dans cette recherche, l'attention s'est essentiellement portée sur deux signifiants, $\mu \hat{v} \theta \circ \varsigma$ et $\lambda o ́ \gamma o \varsigma$, par un privilège directement issu du parcours emblématique mentionné ! Avant d'en faire les marques de deux modes de pensée radicalement opposés, il

sa signification, d'autre part la multiplicité des termes qui en latin peuvent correspondre à notre concept moderne de rite. La théorie étiologique du mythe dérivé du rite/ $\delta \rho \omega \mu \varepsilon v o v$ a été développée par J. HARRISON notamment dans Themis. A Study of the Social Origins of Greek Religion, Cambridge, 1927², p. 327 sq.; on lira ces pages avec le commentaire de H. S. Versnel, What's Sauce for the Goose Is Sauce for the Gander : Myth and Ritual, Old and New, in L. Edmunds (ed.), Approaches to Greek Myth, Baltimore - London, 1990, p. 25-90.

4 Je me suis expliqué sur ces définitions approximatives et nécessairement opératoires du mythe et du rite dans Thésée et l'imaginaire athénien. Légende et culte en Grèce classique, Lausanne, 1990, p. 10 sq. Il est évident qu'en tant que concepts instrumentaux, ni le mythe, ni le rite ne sauraient constituer des réalités, des substances de portée universelle. Il n'y a d'ontologie ni du mythe ni du rite.

5 On songe ici naturellement à l'étude de M. Detienne, L'invention de la mythologie, Paris, 1981, p. 87 sq.; voir aussi P. VEYNE, Les Grecs ont-ils cru à leurs mythes? Essai sur l'imagination constituante, Paris, 1983, qui se centre davantage sur les signifiés et sur les récits pouvant correspondre à notre notion de mythe. 
fallait montrer dans l'évolution du signifié de ces deux termes l'apparition des concepts de mythe entendu comme fiction et de $\lambda$ óyos compris comme discours raisonné. Et même quand la primauté en fut retirée aux Grecs, le mythe est resté l'emblème d'une mentalité primitive opposée au $\lambda$ ó $\gamma$ os de la pensée rationnelle. Mais en anthropologie culturelle et sociale, le mythe c'est avant tout un récit traditionnel; c'est donc cette notion qui nous servira de guide conceptuel.

\subsection{L'usage homérique}

Les aléas des premières transcriptions par écrit des textes de la tradition orale font pour nous de l'œuvre d'Homère le début obligé de toute enquête. Dans les poèmes homériques donc, ce que nous appelons un récit est signifié par deux lexèmes : $\mu \hat{v} \theta$ o et ĕnos. Quand Télémaque demande à Nestor, puis à Ménélas de lui faire le récit de la mort supposée de son père, il s'attend soit à la narration d'un témoin oculaire, soit à un témoignage se fondant sur la transmission orale (aurale, pour être plus précis) des événements. Le récit de ces événements est appelé $\mu \hat{v} \theta 0$ os. Par contre, lorsque le même Télémaque s'enquiert auprès de Nestor du sort réservé à Agamemnon et à Ménélas, il réclame du vieux roi de Pylos un ě́ros; de même les récits d'aède auxquels Eumée compare la narration que lui a faite Ulysse de ses aventures sont-ils désignés par ce

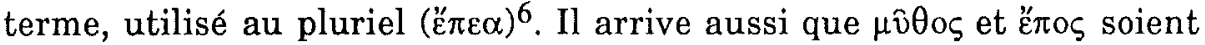
employés de concert, comme de simples doublets. Dans l'Odyssée toujours, les récits qu'adresse à Télémaque Ménélas quand il raconte son retour de Troie et sa rencontre avec Protée sont désignés de ces deux termes, unis sur ce point par un évident rapport de synonymie ${ }^{7}$.

Il va sans dire que les signifiés des deux lexèmes pris en considération en ce début d'étude sont beaucoup plus vastes que ne l'indiquent les quelques exemples mentionnés et s'ils connaissent de nombreux recoupements, ils ne coïncident pas. Il semble que dans les poèmes homériques déjà, ह̌ $\pi$ os désigne davantage la parole dans son aspect substantiel : elle est le réceptacle matériel, susceptible d'assumer plusieurs formes, de différents contenus; les "paroles ailées" (ह̈ $\pi \varepsilon \alpha$ $\pi \tau \varepsilon \rho o ́ \varepsilon v \tau \alpha)$ ont ainsi sur leur destinataire un effet physique. Mv $\theta$ os au contraire dénote plutôt le contenu de la parole, l'avis ou l'intention

6 Hom., Od., III, 94 et IV, 324; III, 243 et XVII, 519, cf. H. Koller, Epos, in Glotta, 50 (1972), p. 16-24; pour l'Iliade, voir G. NAGY, The Best of the Achaeans. Concepts of the Hero in Archaic Greek Poetry, Baltimore - London, 1979, p. $270 \mathrm{sq}$.

7 HoM., Od., IV, 597; cf. également XI, 561. 
exprimés par ce moyen; c'est pourquoi ce lexème peut avoir le sens performatif de 'décision', 'ordre', 'prescription'. Dans un passage célèbre de l'Odyssée, Alcinoos ne compare-t-il pas le récit d'Ulysse à celui d'un aède savant, un récit $\left(\mu \hat{v} \theta \theta_{\varsigma}\right)$ dont l'harmonieuse manifesta-

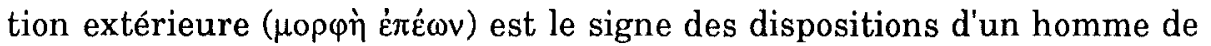
qualité ${ }^{8}$ ?

Mais au-delà de ces valeurs distinctives plus générales propres à $\mu \hat{v} \theta$ o ou à ë $\pi \circ \varsigma$, il faut revenir au récit. Si l'on fait abstraction des verbes

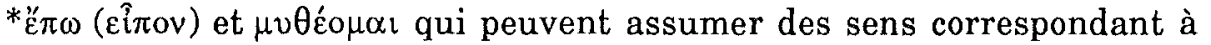
ceux des termes auxquels ils sont morphologiquement apparentés, d'autres termes incluent dans leur signifié le sème de 'récit'. D'abord le

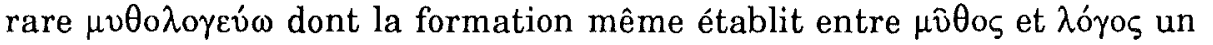
rapport que les modernes devraient juger pour le moins paradoxal! Cet étrange composé ne fait cependant une apparition que dans le passage où il est employé comme synonyme d'un $\mu \nu \theta \hat{\varepsilon} o \mu \alpha \iota$ se référant à l'activité de

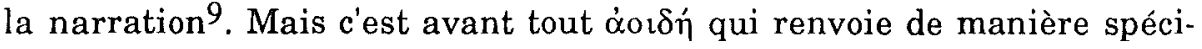
fique au récit de type homérique et que l'on rencontre en concomitance

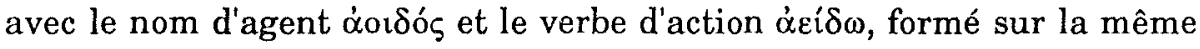
racine, dans tous les passages homériques mettant en scène un aède. Dans l'lliade, Thamyris, l'aède thrace si prétentieux qu'il croit être

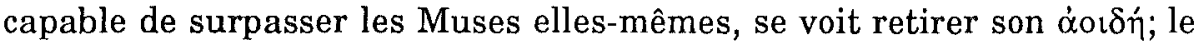
terme englobe ici à la fois la capacité de faiseur de récits de l'aède et le

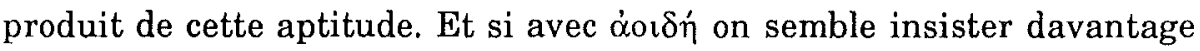
sur l'aspect rythmique et musical du chant épique que sur les paroles dont il est composé, l'ỏotón que chante Phémios à la cour de Pénélope

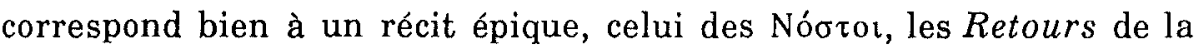
guerre de Troie. Quant au Phéacien Démodocos, son récit rythmé de l'épisode du cheval de Troie, dont l'Odyssée ne donne d'ailleurs qu'un résumé, reçoit naturellement la qualification de $\alpha$ oı $\delta \eta^{10}$.

D'autre part, il est de tradition d'attacher à $\mu \hat{v} \theta 0 \varsigma$ le lexème $\alpha$ îvos. Mais, à côté des sens reconnus de 'fable' que ce terme assume dans les

8 Hom., Od., XI, 367 sq.; cf. H. FouRnIER, Les verbes «dire» en grec ancien, Paris, 1946, p. 211 sq. et 215 sq., ainsi que P. ChantraIne, Dictionnaire étymologique de la langue grecque, Paris, 1968, p. 362 et p. 718 sq., et G.F. GianotTr, Mito e storia nel pensiero greco, Torino, 1976, p. 10 sq. En ce sens, $\mu \hat{v} \theta 0 \varsigma$ désigne des "actes de langage" : cf. G. NAgy, Pindar's Homer. The Lyric Possession of an Epic Past, Baltimore - London, 1990, p. 32 sq.

9 HoM., Od., XII, 450 et 453.

10 HoM., Il., I, 595 et 599; Od., I, 328 et $340 ; O d$., VIII, 498 et 499, cf. également les vers 427 et 580 : ce même récit est désigné par le terme énع $\alpha$ au vers 91 . Sur le sens du terme àoเ $\delta \dot{n}$, cf. encore $L f g r E$, s.v. 
Travaux et les Jours d'Hésiode ou de 'proverbe' comme chez Euripide, il n'y a qu'un emploi où $\alpha i ̂$ ivos désigne réellement un 'récit'. Arrivé à Ithaque, Ulysse tente par la narration d'un épisode inventé de la guerre de Troie d'obtenir d'Eumée son manteau; appréciant ce récit prononcé "selon l'ordre», le porcher cède au héros rusé le vêtement convoité. Le récit a donc ici la finalité utilitaire d'un apologue flatteur, comme c'est le cas de manière encore plus marquée dans les autres passages que les philologues citent à l'appui du sens général, d'ordre performatif, assumé par $\alpha i v o \varsigma^{11}$.

Dans ce contexte, on pourrait encore citer deux lexèmes dont le signifié inclut, mais de manière moins centrale, le sème 'récit'. Il s'agit d'abord de oín, un terme employé métaphoriquement avec le sens de 'chemin du chant'. Les récits de Phémios aussi bien que ceux de Démodocos sont désignés de ce lexème d'un emploi quelque peu marginal; son explication sémantique est à vrai dire sujette à dispute ${ }^{12}$. D'autre part, le terme ü $\mu$ vo l'Odyssée, pratiquement comme synonyme de óoı $\delta$ n. Ce lexème connâ̂tra par la suite une longue fortune comme mot désignant le chant, souvent choral, dans lequel sont retracés les hauts faits des dieux et des héros; il faudra attendre Platon pour que son signifié soit restreint à la prière adressée à un dieu ${ }^{13}$. La mention de ü $\mu v o \varsigma$ et de son signifié pourrait nous entraîner vers la distinction taxinomique que fait le texte homérique entre plusieurs types de chants, première ébauche d'une typologie des genres littéraires. Thrène, hyménée, péan, autant de lexèmes désignant des chants dont le contenu coïncide partiellement avec le récit de hauts faits légendaires, mais dont la spécificité sémantique se réfêre moins à des formes narratives particulières qu'à leur

11 HÉs., Trav., 202; EuR., fr. 508 Nauck $^{2}$; HoM., Od., XIV, 508, cf. Il., XXIII, 652 et 795. Le sens d' 'éloge' est plus fondamental que celui de 'récit' : cf. CHANTRAINE, op. cit. (n. 8), p. 35, et NAGY, op. cit. (n. 6), p. 222 sq. et p. 234 sq; sur d'autres fonctions persuasives de l' $\alpha \hat{\mathfrak{i} v o s}$, voir G. NAGY, Mythe et prose en Grèce archaïque : l'aînos, in C. CALAME (éd.), Métamorphoses du mythe en Grèce antique, Genève, 1988, p. 229-242.

12 Hom., Od., VIII, 481 (cf. aussi 74) et XXII, 347; sur la dispute, on lira J. Svenbro, La parole et le marbre. Aux origines de la poésie grecque, Lund, 1976, p. 36 sq. avec n. 103.

13 Hom., Od., VIII, 429; cf. B. Gentili, Poesia e pubblico nella Grecia antica da Omero al V secolo, Roma - Bari, 1984, p. 48 sq. 
destination; c'est dire que leur signifié renvoie à leurs circonstances d'énonciation respectives ${ }^{14}$.

Faisons donc retour au récit et à sa conceptualisation à travers les termes qui le signifient. "E $\pi$ o $\varsigma$ et $\mu \hat{v} \theta$ o $\varsigma$ désignent donc l'un comme l'autre un récit, mais un récit qui n'est pas forcément valorisé positivement. À côté des récits auxquels on accorde crédit, les poèmes homériques mentionnent des dits trompeurs, destinés à fourvoyer leur destinataire. Pour les dénoter, on utilise aussi bien ërno que $\mu \hat{v} \theta$ os. Au récit plein de ruse qu'Ulysse fait à Polyphème de son prétendu naufrage

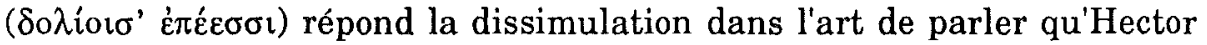

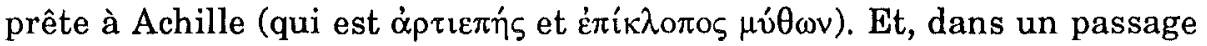
célèbre où la vérité du récit ( $\dot{\alpha} \lambda \eta \theta \dot{\varepsilon} \alpha \quad \mu \nu \theta \eta \dot{\eta} \sigma \sigma \theta \alpha \imath$ ) est opposée au mensonge, Eumée accuse son maître, qu'il n'a pas encore reconnu, de construire une narration ("̈ $\pi \circ \varsigma$ ) pour la seule obtention de l'hospitalité de Pénélope ${ }^{15}$. On constate donc que pour désigner le récit qui n'est digne d'aucune créance, le texte homérique doit préciser l'emploi d'é $\pi$ o $\varsigma$ et de $\mu \hat{v} \theta$ os d'une qualification ou d'un prédicat: pas de 'conte', pas de 'mythe' reconnus comme genre, au sens moderne de ces concepts.

De manière paradoxale, c'est précisément dans l'expression du discours trompeur et mensonger que l'on trouve l'un des rares emplois homériques du terme de $\lambda$ ó $\gamma$ os. C'est en effet par des discours rusés

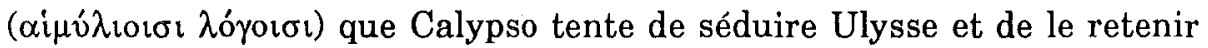
auprès d'elle. Et, peut-être à la même époque, Hésiode couple

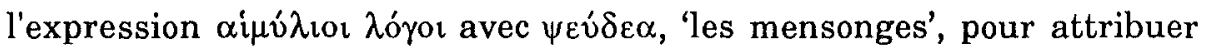
ces paroles insidieuses comme parure de séduction à Pandora; c'est par des mots séducteurs analogues que Zeus trompe Métis pour mieux l'engloutir ${ }^{16}$. Mais les textes du poète d'Ascra - faut-il y voir

14 Sur cette question, voir mes Réflexions sur les genres littéraires en Grèce archaique, in QUCC, 17 (1974), p. 113-128, ainsi que R.L. FOwLER, The Nature of Early Greck Lyric : Three Preliminary Studies, Toronto - London, 1987, p. 86 sq.

15 Comparer Hom., Od., IX, 282 à $I l$., XXII, 281; cf. aussi Od., XIV, 124 sq. Sur d'autres termes également utilisés dans la poésie homérique pour désigner les discours mensongers, on verra W. LUTHER, "Wahrheit» und "Lilge» im ältesten Griechentum, Borna - Leipzig, 1935, p. 70 sq.

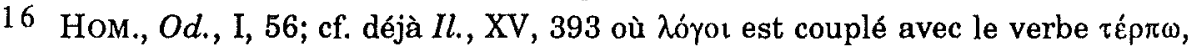
«charmer». HÉs., Trav., 78; voir aussi le vers 789 (les paroles séductrices étant dans ce passage l'attribut d'un garçon !); HÉs., Théog., 890. On se souviendra qu'HésIoDE (Théog., 229) fait des $\Psi \varepsilon v \delta \varepsilon i \varsigma \zeta \Lambda o ́ \gamma o ı$ personnifiés les enfants d'Éris,

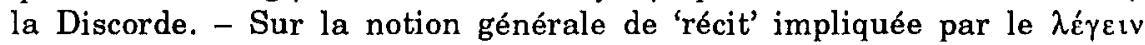

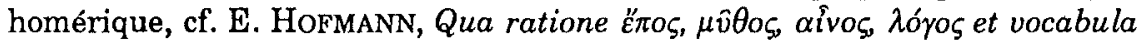


l'influence de l'utilisation progressive de l'écriture ? - marquent pour nous un point de non-retour. En faisant dire aux Muses, à l'occasion du célèbre prologue de la Théogonie, la possibilité de raconter ( $\lambda e^{\prime} \gamma \varepsilon \imath v$ ) des mensonges tout pareils à la réalité, Hésiode introduit le doute dans l'énonciation du récit épique lui-même ${ }^{17}$. La séduction et la tromperie semblaient écartées de la narration énoncée par l'aède inspiré par les Muses; avec Hésiode, tout récit peut devenir mensonge. Aucune catégorie spécifique, avec un signifiant correspondant, n'est donc encore réservée au récit considéré comme fiction.

\subsection{Les critiques du $\lambda o ́$ os}

Dans le domaine de l'expression de l'énoncé sous toutes ses formes, les lexèmes étudiés jusqu'ici assument donc dès Homère des sens qui sont loin de se limiter à ce que nous entendons sous le terme de récit. En plus de son sens narratif déjà indiqué, relatif à la matérialité du discours, ह̌лo sive pendant le combat jusqu'aux prophéties de Tirésias en passant par

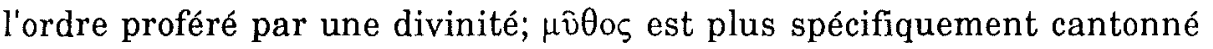
dans la désignation du discours qui exprime une pensée, un avis, un dessein, quand il n'a pas le sens performatif mentionné plus haut ${ }^{18}$. Mais la mise en cause, par Hésiode, de la véracité du discours épique nous invite à passer à la dimension diachronique en concentrant l'analyse sur le champ sémantique plus restreint recouvert par la notion de récit mis en forme par un homme de métier, avant d'examiner si la vraisemblance de ce récit constitue un critère de délimitation comme cela semble être le cas pour le concept moderne de mythe.

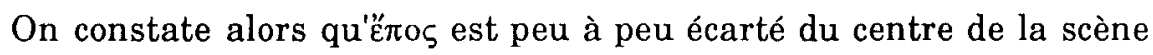
lexicographique pour acquérir des valeurs sémantiques très spéci-

$a b$ eisdem stipibus derivata in antiquo Graecorum sermone (usque ad annum fere 400) adhibita sunt, Göttingen, 1922, p. 103 sq.

17 Hés., Théog., 26 sq. P. Puccr, Hesiod and the Language of Poetry, Baltimore London, 1977, p. 8 sq., montre que dans cette perspective le $\psi \varepsilon \hat{v} \delta$ o $\zeta$ et l' $\dot{\alpha} \lambda \dot{\eta} \theta \varepsilon \imath$ dépendent tous deux du $\lambda$ ó ${ }^{\circ} \varsigma$, sans que le premier renvoie à un genre particulier, comme l'ont pensé la plupart des interprètes de ce passage; voir par exemple H. NeItzel, Hesiod und die lügenden Musen, in Hermes, 108 (1980), p. 387-401, avec les remarques que j'ai présentées à ce propos dans Le récit en Grèce ancienne. Énonciations et représentations de poètes, Paris, 1986, p. 57 sq. - C'est le lieu de rappeler ici le fameux adage de Solon, fr. 25 Gentili-Prato : "Les aèdes racontent beaucoup de mensonges"!

18 HoM., Il., III, 83; Od., XII, 266; Il., I, 216 et VIII, 8; Od., VII, 157 et I, 358. 
fiques. C'est essentiellement l'usage d'Hérodote qui marque pour nous ce changement; ह̌ $\pi$ o devient chez l'historien d'Halicarnasse d'une part le terme qui désigne le mot comme unité linguistique et d'autre part, employé au pluriel, le lexème désignant spécifiquement le poème épique. Cette première délimitation dans la langue de la poésie épique comme genre littéraire est à vrai dire déjà esquissée chez les poètes méliques où, dès Alcman, on dissocie dans la poésie les paroles de la composante musicale. "E $\pi \varepsilon \alpha$ assume dès lors le sens de 'vers' méliques ou épiques; on trouve cette spécification dans la dénomination du discours poétique par exemple chez Pindare ${ }^{19}$. Le retrait du terme ع̈ $\pi$ $\varsigma$ dans des emplois particuliers laisse la place libre à $\lambda o ́ \gamma \circ \varsigma$, un lexème polysémique s'il en est. D'un emploi restreint dans la poésie homérique, il va peu à peu reprendre toutes les fonctions signifiantes de ع̈ros : 'énoncé', 'discours', 'discussion', mais aussi 'prophétie' ou 'maxime'. Dans le domaine du récit, son très vaste champ d'application dénote encore la grande indifférenciation qui règne sur la représentation du narratif : pas de typologie du récit, pas de taxinomie de la narration.

Ainsi chez Hérodote l'absence de nomenclature du récit est assortie de la seule exception que représente le récit de forme épique désigné spécifiquement du terme $\ddot{\varepsilon} \pi \varepsilon \alpha$. Que le récit épique soit attribué à Homère, au fabuleux Aristée de Proconnèse ou qu'il soit anonyme comme les Cypria, peu importe; Homère aussi bien qu'Aristée sont des $\dot{\varepsilon} \pi$ лoroí, des faiseurs de récits qui détiennent l'art de mettre en vers

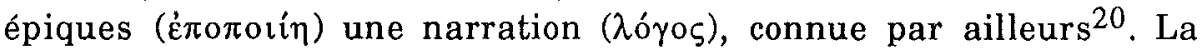
distinction induite par l'emploi de "̈л $\varepsilon \alpha$ porte donc essentiellement sur la forme du discours. Mais tout récit, quel qu'en soit le contenu, reste de l'ordre du $\lambda$ óyos. Ainsi les récits légendaires qu'Hécatée recueille, des récits qui dans leur multiplicité peuvent prêter à rire, sont tous des $\lambda$ óyor; et $\lambda$ ó $\gamma \circ \varsigma$ est aussi l'ensemble du récit d'Hérodote. Ce récit, son auditeur ou son lecteur, de l'aveu même de son auteur, n'est pas contraint à y accorder crédit : Hérodote rapporte par l'écriture ce qu'il a entendu quand ce n'est pas ce dont il a été le témoin oculaire. Mais il ne se sent pas l'obligation de critiquer systématiquement ses sources. Il se contente la plupart du temps de transcrire tels quels les $\lambda \varepsilon \gamma o ́ \mu \varepsilon v \alpha$, les dits

19 Alcman, fr. 27 et fr. 39 Page, avec le commentaire que j'en ai proposé dans Alcman, Roma, 1983, p. 463 sq. et p. 480 sq.; PIND., Ol., III, 8, Ném., IX, 3 et II, 1

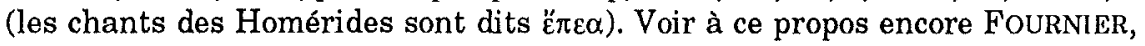
op. cit. (n. 8), p. 214 sq.

20 HDT., II, 116 et IV, 29; IV, 13, 1; 14, 3 et 16, 1; II, 117; غ̇лотоเ́́ : VII, 161, 3, cf. II,

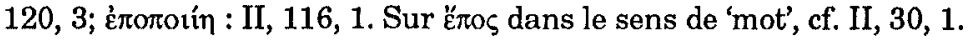


des autres, ces spécialistes de la narration ( $\lambda$ ó $\gamma$ เo $\alpha \ddot{\alpha} \delta \rho \varepsilon \varsigma$ ) qu'il a régulièrement consultés et écoutés dans les différentes étapes de son enquête, que leurs récits soient $\pi \imath \alpha v o i$, dignes de confiance, ou non.

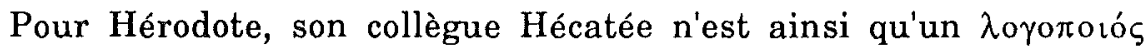
parmi d'autres; l'historien ne réserve aucun terme spécifique à la désignation du récit véridique ou au contraire du récit mensonger ${ }^{21}$. On notera à ce propos que chez Pindare, les aèdes eux-mêmes, auteurs de poèmes épiques, n'apparaissent qu'inclus dans la catégorie plus générale des $\lambda$ ó yıo, des raconteurs de récits, seuls détenteurs du souvenir attaché à la valeur des hommes qui ne sont plus, et par conséquent propagateurs du $\kappa \lambda \varepsilon_{\text {ó }}{ }^{22}$.

Quant à Xénophane, le pourfendeur d'une tradition qui devient mythologique au sens où nous l'entendons aujourd'hui, il ne dispose pas non plus d'un terme technique apte à définir les récits qui la constituent et qu'il critique. Ces récits, il les désigne par leurs sujets - combats des Titans, des Géants et des Centaures - et il oppose ces "fictions des anciens" ( $\left.\pi \lambda \alpha \dot{\alpha} \sigma \mu \alpha \tau \alpha \tau \hat{\omega} v \pi \rho \tau^{\prime} \rho \omega v\right)$ aux éloges qu'il convient d'adresser

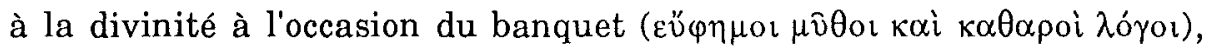
'paroles pieuses et récits purs'.

La critique adressée par Xénophane aux poèmes homériques et hésiodiques ne s'adresse donc pas à un genre, encore moins à un genre qui réunirait tous les récits désormais taxés de fiction; ces récits, ce sont des $\lambda$ óyor comme sont aussi des $\lambda$ ó yor les compositions de Xénophane luimême. Et s'ils sont l'objet d'un rejet, ce n'est pas pour des raisons d'invraisemblance théologique, mais, le cas échéant, parce qu'ils sont jugés selon un critère social d'utilité dans le cadre de la cité 23 . Il s'agit donc encore d'un oui, mais d'un oui conditionnel à la tradition; on la

21 HÉCatéE, 1 F 1a Jacoby; HDT., II, 123, 1 et VII, 152, 3, cf. aussi le célèbre passage de II, 99, 1 à propos de l'enquête sur l'Égypte; $\lambda$ ó́loı: I, 1, 1; II, 3, 1; IV, 46 , 1, etc.; sur HÉCATÉE lui-même : II, 143, 1; V, 36, 2; V, 125. F. JACOBY, art. Herodotos, in $R E$ Suppl. II (1913), col. 206-519 (col. 396 sq.), a dressé le répertoire complet des indications que donne HÉRODOTE sur les $\lambda$ ó yor qui se disent ( $\lambda \dot{\varepsilon} \gamma_{\text {yov } \tau \alpha \mathfrak{l})}$ dans son cuuve de transcription; on verra à ce propos les commentaires de F. HARTOG, Le miroir d'Hérodote. Essai sur la représentation de l'autre, Paris, 1980, p. 282 sq., CALAME, op. cit. (n. 17), p. 73 sq., et C. DARBOPEschanski, Le discours du particulier. Essai sur l'enquête hérodotéenne, Paris, 1987, p. 113 sq.

22 PIND., Pyth., I, 93 sq.; Ném., VI, 45 sq. et 28 sq., avec les remarques de G. NAGY, Herodotus the logios, in Arethusa, 20 (1987), p. 175-184.

23 Xénophane, fr. 1, 13 sq. et fr. 6, 1 Gentili-Prato. Voir à ce propos Detienne, op. cit. (n. 5), p. 123 sq., et surtout D. BABUT, Xénophane critique des poètes, in $A C$, 43 (1972), p. 83-117. 
respectera pour autant que les actions qu'elle met en scène ne soient pas marquées du sceau de la démesure. Car la tradition, pour être retenue, doit servir les intérêts des citoyens.

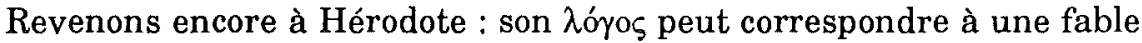
comme celle du joueur de flûte que Cyrus raconte en guise de réponse à la demande de soumission des Ioniens et des Éoliens. On pourrait même se surprendre à espérer qu'accompagné du qualificatif įpós, le $\lambda$ ó faut cependant vite déchanter puisque l'utilisation de l'expression iєpò $\lambda$ ó $\gamma$ os est limitée chez Hérodote au récit égyptien. Par contre, plus avant dans le texte, la légende à valeur explicative et fondatrice des Héraclides reçoit le statut d'un simple $\lambda$ ó $\gamma \circ \varsigma$, sans que lui soit attribuée la moinơre valeur sacrée : chez Hérodote non plus, le mythe au sens moderne du terme n'est pas élevé au rang de catégorie narrative. Et quand le récit comporte deux versions, il devient tout simplement, avant même l'intervention des Sophistes, un $\delta$ r ò $_{\text {c }} \lambda$ ó$_{0} \varsigma$, un 'récit double'. De même en va-t-il des différentes versions du «mythe» du rapt d'Hélène : épiques ou non, ce sont autant de $\lambda$ ó$_{0 .}{ }^{24}$.

Pourtant, plus d'un siècle avant Hérodote, Stésichore, le citharode

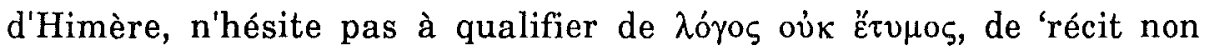
véridique', précisément la version de la légende d'Hélène qu'il a traitée dans un précédent poème. Et c'est aussi un poète mélique, Pindare, qui se met à distinguer dans la tradition les récits qu'il faut retenir et au contraire ceux qui ne sont bons qu'à être rejetés. Soit à propos de la légende du cannibalisme de Tantale accusé d'avoir dévoré les membres bouillis de son fils Pélops, soit au sujet de la renommée exagérée dont jouit Ulysse grâce aux "douces paroles d'Homère», Pindare pour la première fois attribue à $\mu \hat{v} \theta$ os une valeur négative, réservant au $\lambda$ óyos l'expression de la vérité 25 : les $\mu \hat{\theta} \theta$ o , tissus de mensonges, sont trompeurs d'hommes; ils parviennent à les aveugler grâce aux machineries charmeuses de l'art poétique. D'un côté donc l'à $\lambda \eta \theta \eta_{\eta} \varsigma \lambda{ }^{\prime} \gamma \circ \varsigma$, le

24 HDT., I, 141, 1; II, 147, 2; 51, 4; 62, 2 et 81, 2, à comparer avec IX, 26, 3. Voir aussi III, 32, 1 et II, 116, 1 sq. sur les différentes versions de la légende d'Hélène.

25 StÉsıch., fr. 192, 1 Page. PIND., Ol., I, 27 sq. et Ném., VII, 22 sq.; cf. aussi Ném., VIII, 33 et V, 14 sq.; mais il faut relever que dans les Ol., VII, 20 sq. et IX, 35 sq., c'est le terme $\lambda$ ó ${ }^{\prime} \varsigma$ qui dénote un récit "mythique» corrigé ou rejeté par le poète! Commentaire et bibliographie à ce sujet chez D. E. GERBER, Pindar's Olympian One : A Commentary, Toronto - London, 1982 (Phonix Suppl. 15), p. 62 sq., et chez A. KOMORNICKA, L'attitude de Pindare envers les poètes et son reflet chez Platon, in Actes du VIIe Congrès de la F. I. E. C. I, Budapest, 1983, p. 199-205. 
discours véridique; de l'autre, les $\mu \hat{v} \theta$ or mensongers. Le partage séman-

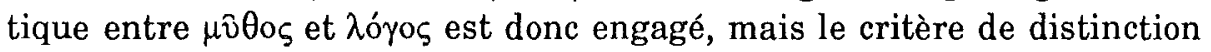
s'appuye sur une conception très particulière de la vérité; elle ne fait pas référence à un vraisemblable qui se fonderait sur la confrontation du contenu du récit avec la réalité et sur son adéquation avec un référent extra-linguistique. Le critère de la vraisemblance est ici d'ordre éthique : est vrai ce qui correspond à certaines règles de comportement. L'établissement de la vérité repose ainsi sur un jugement d'ordre moral. Conditionnée par une norme à la fois sociale et individuelle, la distinction entre le vrai et le faux chez Pindare ne correspond donc pas à la moindre esquisse d'une typologie du récit; il y a simplement des légendes qui sont dignes d'être reprises par le poète et d'autres qui doivent être condamnées à l'oubli.

Certes, les deux uniques emplois hérodotéens du terme $\mu \hat{v} \theta$ os sont relatifs à des récits dont l'historien met précisément en cause la valeur de vérité. Dans un premier cas est qualifié de $\mu \hat{v} \theta$ o le récit des Grecs ( $\lambda \varepsilon ́$ ́vovoı !) rapportant comment Héraclès aurait échappé au sacrifice humain auquel le destinaient les Égyptiens; récit condamné au nom de l'inexpérience des Grecs vis-à-vis de la nature et des coutumes des

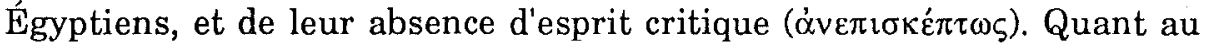
second emploi de $\mu \hat{v} \theta$ o $\varsigma$ chez Hérodote, il correspond à la désignation de cette version de l'explication des crues du Nil qui suppose l'existence du fleuve Océan; par manque de preuves, l'existence d'Océan doit être considérée comme poétique et fictive. Mais dans ces deux cas, le caractère de "mythe" du récit concerné est précisé par une qualification faisant référence à sa naïveté ou à son obscurité. Môlos ne désigne donc pas chez Hérodote un type particulier de récit; et une analyse qui abandonnerait les signifiants grecs pour se concentrer sur les signifiés non plus des lexèmes, mais des énoncés narratifs - montrerait que pour un Hérodote il n'y a pas de différence qualitative entre les événements qui pour nous appartiennent à la légende et ceux que nous classerions du

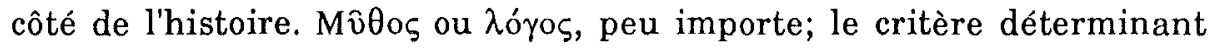

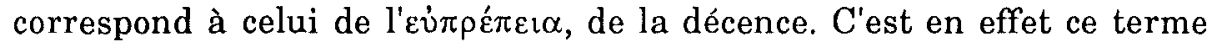
qui qualifie chez Hérodote la version ( $\lambda$ ó $\gamma \circ \varsigma$ ) de la légende d'Hélène finalement retenue par Homère ${ }^{26}$.

26 HDT., II, 45, 1 et 23; sur le premier passage, on lira M. PIÉRART, L'historien ancien face aux mythes et aux légendes, in $E C, 51$ (1983), p. 47-62, qui montre à cette occasion combien ce que nous considérons comme légende passe aux yeux des Grecs pour faire partie intégrante de leur passé historique; voir aussi les remarques de M.I. FinLey, The Use and Abuse of History, New York, 1972, p. 11 sq., et celles, à venir, de S. SAïD dans l'étude citée infra n. 33. Sophismes à 


\subsection{Les philosophes et la véridiction}

Il faut attendre Platon, certainement dépendant en cela de la tradition sophistique, pour trouver quelques-uns des présupposés de la notion moderne du mythe; cette catégorie commence alors à prendre davantage de consistance que celle qu'on a consenti à lui attribuer. Fondés ou non, des critères de distinction au sein des productions narratives sont désormais établis : par le médium du $\lambda$ ó $\gamma \circ \varsigma$, et par analogie avec la peinture, le mythe devient l'image discursive des dieux et des héros 27 . De là des termes nouveaux comme $\mu v \theta 0 \lambda$ ó $\eta \mu \alpha$,

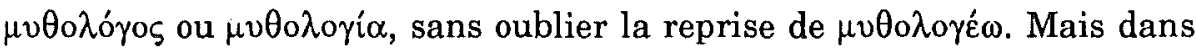
la critique que le philosophe adresse à ces simulacres narratifs, ce n'est pas l'existence des dieux et des héros qui est mise en doute. L'organisateur de la Cité idéale adopte à cet égard une position analogue à celle de Pindare : ce sont Homère, Hésiode et leurs successeurs qui ont raconté des $\mu \hat{v} \theta 0 \iota \psi \varepsilon v \delta \varepsilon \hat{\imath} \zeta$; et ces récits "mensongers» ne sont pas condamnables parce qu'ils ne correspondraient pas à une vérité factuelle, mais ils le sont parce qu'ils sont considérés comme "mauvais» $(\chi \alpha \lambda \varepsilon \pi \circ i)$. Sans doute les actes répréhensibles et d'ailleurs innommables qu'un Hésiode prête à Ouranos et à ses successeurs pourraient correspondre à une vérité $(\dot{\alpha} \lambda \eta \theta \hat{\eta})$. Là n'est pas la question; l'essentiel, si on n'en réserve pas le récit à une minorité d'initiés, c'est de les taire!

Ces récits sont néfastes en ce qu'ils donnent de la divinité une image qui ne correspond pas à son essence : le Bien. Acceptons donc au sein de la Cité les faiseurs de mythes ( $\mu v \theta$ oлo ói), en général des poètes

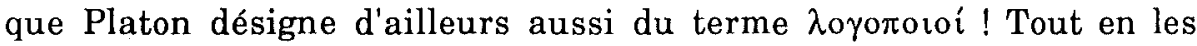
soumettant à la censure : seuls leurs ubeaux" récits seront retenus. Thésée lui-même et ses actes de rapt violent n'échapperont donc pas aux censeurs de la Cité. Il y a ainsi des choses qui ne sont pas bonnes à être narrées et ce sera donc le rôle des fondateurs de la Cité de faire que seuls

ce propos chez HARToG, op. cit. (n. 21), p. 303 sq. Ce n'est que Diodore DE SICILE $(I, 37,1)$ qui interprétera les explications des crues du Nil comme

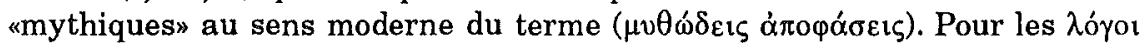
sur Hélène, voir HDT. II, 116, 1. - Chez ARISTOPHANE encore, le terme $\mu \hat{v} \theta$ os a besoin d'une qualification spécifique quand il renvoie à un récit auquel on ne

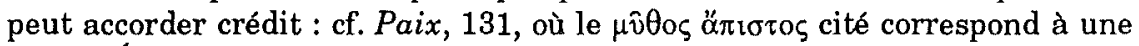
fable d'Ésope ! Voir également l'emploi du mot dans les Guêpes, 1179, avec le commentaire de L. EDMUNDs, Introduction : The Practice of Greek Mythology, in op. cit. (n. 3), p. 1-20.

27 Platon, Rép., 377e : c'est notamment Detienne, op. cit. (n. 5), p. 155 sq., qui a tenté de couper la *mythologie" platonicienne de notre concept de mythe. 
les récits $(\mu \varepsilon \mu v \theta \circ \lambda \circ \gamma \eta \mu \varepsilon ́ v \alpha)$ susceptibles de conduire à la vertu parviennent aux oreilles des jeunes. Aux poètes la composition des récits

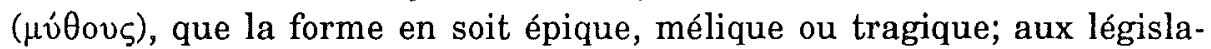

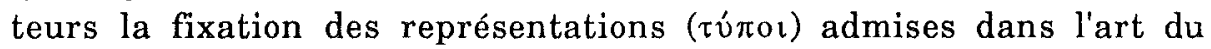

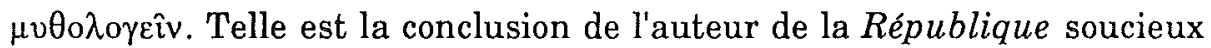
de préserver la Cité des fables amorales de la poésie ${ }^{28}$. Est donc en cause l'exemple de comportement que peut fournir le récit traditionnel, et non pas sa valeur de vérité. De même au début du Phèdre, Socrate se refuse-til à mettre en doute la vraisemblance ( mais aussi $\lambda$ ó ${ }^{\circ} \varsigma$ !) de l'enlèvement d'Oréithyia par Borée. Il préfère accorder crédit à la tradition ( $\tau$ ò $v o \mu \iota \zeta o ́ \mu \varepsilon v o v$ ) : peu importe sa valeur de vérité 29 !

Création de néologismes et tentatives explicites de définition, autant d'éléments permettant d'identifier la naissance probable d'une nouvelle catégorie. Mais la distinction du mythe d'avec d'autres types de discours ne se fait pas au nom de la véracité quant aux faits narrés. Assurément, dans certains de ses emplois, le mot $\lambda$ ó ${ }^{\circ}$ s semble dénoter par contraste un discours qui, argumenté, peut aussi être l'objet d'une vérification factuelle. Et l'enquête menée par Brisson montre bien l'existence chez Platon d'une délimitation consciente entre un discours argumentatif et vérifiable, et un mythe incluant les récits dont le contenu, confié à la mémoire sous forme volontiers poétique, ne peut être l'objet d'une vérification ${ }^{30}$. De fait, dans deux développements en tout cas, Platon oppose explicitement le $\mu \hat{v} \theta 0 \varsigma$, ou de manière plus ambiguë les

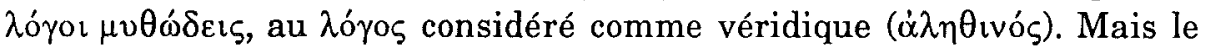
$\lambda$ ó $\varsigma \varsigma$ de vérité renvoie dans le premier cas au grand «mythe» de l'Atlantide et à sa réalisation possible dans l'histoire! Dans le second

28 Platon, Rép., 377d-380c; cf. également 391c sq. où sont condamnés tous ces récits qui font des héros des êtres aussi mauvais que les hommes; en 392a sont désignés du terme hóyot les récits qui, se référant aux dieux, aux démons, aux héros et aux êtres de l'Hadès, doivent être tenus sous le contrôle des fondateurs de la Cité. Voir aussi, dans le même sens, Lois, 663d sq. Sur l'emploi de

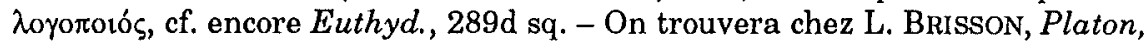
les mots et les mythes, Paris, 1982, p. 177 sq. et p. 184 sq., une bonne analyse sémantique des différents composés de $\mu \hat{v} \theta$ os dans leurs emplois chez PlatoN.

29 Platon, Phèdre, $229 \mathrm{~b}$ sq. On remarquera encore que dans le Phédon, $61 \mathrm{~b}$, les $\mu \hat{v} \theta 0$ correspondent aux fables ésopiques mises en vers par Socrate dans ses exercices poétiques.

30 BRISSON, op. cit. (n. 28), p. 111 sq. 
cas, il se réfère à la composante verbale de la musique ${ }^{31}$. Dans cette conception en effet, les récits qui assument une forme poétique et par conséquent musicale ne sont pas forcément de nature "mythique", ils peuvent aussi correspondre à une certaine vérité "historique", sinon factuelle. La frontière ne passe donc pas entre un $\mu \hat{v} \theta 0 \varsigma$ de fiction et un $\lambda$ ó yos de raison. Mais, sans qu'elle soit marquée dans l'utilisation de signifiants tout à fait distincts, la limite définie par Platon se fonde sur le critère de l'utilité. Tant pis si certains récits qui se réfêrent au passé légendaire de la cité et qui sont en général désignés comme $\mu \hat{v} \theta 0 \imath$ ne peuvent être l'objet d'une vérification; ce qui compte, c'est que, comme les $\lambda$ ó ${ }^{\circ}$ i argumentés et vérifiables, ces récits puissent servir les objectifs élevés assignés à l'éducation dans la Cité idéale ${ }^{32}$.

À la même époque on trouve d'ailleurs sous les emplois que font les orateurs du terme $\mu \hat{v} \theta$ os une conception proche de celle de Platon; exception faite naturellement de la réflexion du philosophe sur le récit comme simulacre d'une réalité à son tour simple simulacre de l'essence des choses. Ainsi Démosthène classe-t-il dans la catégorie du $\mu \hat{v} \theta$ o $\zeta$ ces récits héroïques, tels l'Amazonomachie, la guerre menée par Eumolpe contre les Athéniens ou l'accueil réservé à Adraste par Thésée à Athènes, que l'on cite volontiers dans une oraison funèbre. Ces récits, ils peuvent connaître différentes versions ( $\lambda$ ó $\gamma \circ$ ) et des mises en forme épiques, méliques ou même prosaïques. Le $\mu \hat{v} \theta$ os est donc référé ici à un contenu, à son contenu héroïque, en contraste avec les actions plus proches dans le temps qui, quant à elles, n'ont pas encore pu bénéficier du processus de "mythologisation" (ơ̈ $\pi \omega \mu \varepsilon \mu v \theta o \lambda o ́ \gamma \eta \tau \alpha \imath)$ ! Dans une perspective analogue, Isocrate réfêre les $\mu \hat{v} \theta$ or aux luttes et aux guerres des demi-dieux : Homère les a transformés en récit "mythologique»

31 Platon, Timée, 26e (cf. 29d) et Rép., 522a. J.-P. VERnant, Mythe et société en Grèce ancienne, Paris, 1974, p. 200 sq., voit encore dans ces deux passages une

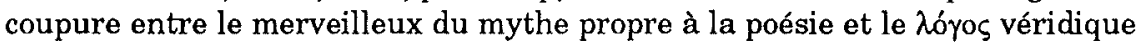
élaboré par les philosophes. Voir à ce propos le commentaire de M. DETIEnNe, La double écriture de la mythologie entre le Timée et le Critias, in CALAME (éd.), op. cit. (n. 11), p. 17-33 (repris dans L'écriture d'Orphée, Paris, 1989, p. 167 186). Ce sont les commentateurs de Platon qui poseront en termes modernes la question de la vérité historique du «mythe» de l'Atlandide : cf. Proclus, In Platonis Timaeum, 20d (I, p. 75, 30 sq. Diehl). - Une seule fois chez Platon, dans le Protagoras, 324d, la légende (il s'agit du mythe de Prométhée : cf. 320c)

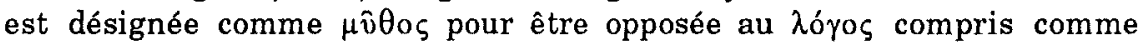
discours philosophique fondé sur le raisonnement; mais l'une et l'autre sont mis au service de la démonstration ( $\dot{\varepsilon} \pi\llcorner\varepsilon \varepsilon \hat{\xi} \xi \omega)$ sur l'essence de la vertu : cf. BRISSON, op. cit. (n. 28), p. 139 sq.

32 Voir notamment Platon, Rép., 382 c-d, 398a-b; Lois, 663d-e, etc. 
( $\dot{\varepsilon} \mu v \theta 0 \lambda o ́ \gamma \eta \sigma \varepsilon v)$ tandis que les Tragiques les ont transposés en actions scéniques, faisant de leur public non plus des auditeurs, mais des spectateurs. À travers le terme de $\mu \hat{v} \theta_{\text {os }}$, ce qui est maintenant conceptualisé c'est une distinction entre une histoire ancienne, héroïque, et une histoire moderne, encore non "mythologisée»33. Mais il n'est pas question de mettre en doute la valeur de vérité des récits tombant dans la catégorie du $\mu \hat{v} \theta$ os : pas d'opposition donc entre la fiction du mythe et la vérité de l'histoire, pas d'hétérogénéité fondamentale entre le légendaire et l'historique. Tout au plus Isocrate met-il en doute, peut-être à la suite de Thucydide, l'utilité des mythes qui entrent dans la catégorie plus générale de ces $\lambda$ ó $\gamma o t$ dont le but est de charmer leur auditoire. Dans cette fonction, la forme poétique a une place privilégiée et la légende garde volontiers sa valeur paradigmatique.

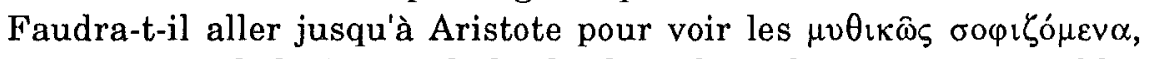
les «arguties mythologiques» de la théologie hésiodique rejetées en bloc au profit du raisonnement argumentatif ( $\left.\tau \dot{\alpha} \delta \imath^{\prime} \dot{\alpha} \pi \circ \delta \varepsilon \varepsilon^{\prime} \xi \varepsilon \omega \zeta \lambda \varepsilon \varepsilon^{\prime} \gamma \circ \tau \varepsilon \varsigma\right)$, pour assister donc enfin à une opposition nette entre un mythe héroïque de fiction et un discours construisant sa vérité sur la raison? Illusion partielle puisque dans ce passage de la Métaphysique, comme dans un passage parallèle de l'Histoire des animaux, $\mu \hat{v} \theta$ os et son dérivé ne renvoient pas au récit, mais à une allégation physique ou biologique sans fondement; illusion aussi puisque $\mu \hat{v} \theta$ os dans son sens narratif désigne essentiellement chez Aristote l'intrigue d'un récit, indépendamment de sa véracité ${ }^{34}$.

33 DÉmosth., Epit., 8 sq.; IsocR., Nicoclès, II, 48 sq. Je ne tire ici que deux exemples du dossier beaucoup plus riche présenté par S. SAï sous le titre De l'histoire au mythe: l'exemple des guerres médiques dans la rhétorique au cours de 3e cycle Ecritures du mythe et de l'histoire en Grèce et à Rome (Les Diablerets, 4-9.3.90) et qui sera publié dans un proche avenir. - Sur l'absence chez les Grecs classiques de la notion moderne de vérité historique, cf. VEYNE, op. cit. (n. 5), p. 69 sq.; sur l'absence concomitante de distinction tranchée du point de vue de la réalité des faits entre légende et histoire, voir supra n. 26.

34 ARISTOTe, Métaph., 1000a 5 sq., et Hist. anim., 578 b 24 sq.; cf. aussi $579 \mathrm{~b} 4$ sq. et $580 \mathrm{a} 14 \mathrm{sq}$. où $\mu \hat{v} \theta$ os signifie conjointement l'allégation biologique et le récit légendaire. Sur la polysémie du terme $\mu \hat{v} \theta 0 \varsigma$ dans son emploi aristotélicien, voir en dernier lieu V. CEssi, Praxis e Mythos nella Poetica di Aristotele, in QUCC, 48 (1985), p. 45-60, et les remarques que j'ai présentées dans Illusions de la mythologie, à paraître. - Le sens technique d'intrigue' qu'ArIsTơ attribue à $\mu \hat{v} \theta$ os dans la Poétique (voir notamment 1450a 4 sq.) montre l'aspect mis en valeur dans la signification narrative de ce terme : voir à ce propos P. RICCEUR, Temps et récit. Tome I, Paris, 1983, p. 57 sq. 
Il est donc finalement bien isolé dans la tradition historiographique de la fin du Ve siècle, ce Thucydide qui dit se défier aussi bien des récits des poètes que de ceux de ses prédécesseurs les logographes : récits rejetés non pas pour leur caractère amoral, mais par insuffisance de preuves, parce que ce sont des récits avant tout destinés à charmer des oreilles attentives à une tradition essentiellement orale. L'invérifiable, l'imprédictible, et finalement l'utile sacrifié sur l'autel du discours charmeur, tels sont les critères qui définissent le $\mu v \theta \omega \hat{\omega} \delta \varepsilon \varsigma$ en opposition à la narration de l'historien qui se fonde sur des témoignages oculaires ${ }^{35}$. Mais le $\mu v \theta \hat{\omega} \delta \varepsilon \varsigma$ n'est qu'une qualification délimitant une classe extensible de récits très divers; il ne constitue pas un genre narratif.

De manière significative, cinq siècles plus tard, quand Plutarque tente de définir préalablement les caractères discursifs et énonciatifs de son récit biographique sur Thésée, les genres à peine esquissés sont à nouveau confondus. C'est que Plutarque nous propose une "archéologie», et ces anciennes histoires, même quand ce qu'elles ont de "mythique» $(\mu \nu \theta \hat{\omega} \delta \varepsilon \varsigma)$ a pu être soumis à l'œuvre de la raison ( $\left.\lambda o^{\prime} \gamma_{\circ}\right)$, conservent des éléments contraires à la vraisemblance factuelle ${ }^{36}$. Voilà donc enfin le "mythique" explicitement entendu dans l'une de ses connotations modernes, celle de l'invraisemblable et de l'incroyable; mais c'est pour en brouiller immédiatement les contours : d'une part en employant un peu plus loin le terme de $\mu \hat{v} \theta$ os avec le simple

35 Thuc., I, 21, 1 et 22, 4, avec le commentaire de B. Gentili et G. Cerri, Storia e biografia nel pensiero antico, Roma - Bari, $1983^{2}$, p. 10 sq., et celui de Detienne, op. cit. (n. 5), p. 105 sq. Chez Isocrate (Panath., 1 sq.), les גóyoı $\mu v \theta \omega ́ \delta \varepsilon เ \varsigma$, s'ils coïncident bien avec des récits fabuleux et pleins de mensonges

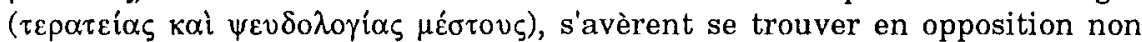
pas avec des discours véridiques, mais avec des discours qui visent avant tout à servir les intérêts de la cité : voilà donc à nouveau le $\sigma v \mu \varphi \varepsilon ́ p o v$ au centre du discours avrai"!

36 Plut., Thésée, 1, 5 et 15, 2. Certes, au siècle précédent, DenYs d'HaliCARNASSE (Thuc., 7) décèle dans les histoires des historiens classiques des $\mu \hat{\theta} \theta 0$ entendus comme récits fabuleux (cf. Detienne, op. cit. [n. 5], p. 145 sq.), mais DenYS n'hésite pas à retenir dans son histoire de Rome des récits qu'il gratifie de

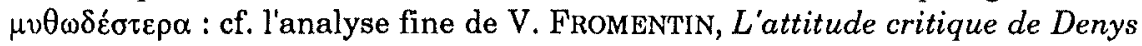
d'Halicarnasse face aux mythes, in $B A G B, 47$ (1988), p. 318-329. Et un siècle plus tard, SEXTUS EMPIRICUS, Adv. gramm., 263 sq., distinguera entre le $\pi \lambda \alpha \dot{\alpha} \sigma \alpha$, discours vraisemblable, le $\mu \hat{v} \theta$ os, discours mensonger sur ce qui ne s'est point passé, et l'ioropía, exposition de ce qui s'est réellement passé. Mais cette conscience se mêle avec l'exigence d'un nouveau "merveilleux" qui prend les apparences de l'histoire : cf. VEYNE, op. cit. (n. 5), p. 57 sq., et l'étude à paraitre citée n. 34 . 
sens de 'version narrative', indépendamment de tout jugement sur la valeur de vérité de cette version; d'autre part en requérant du lecteur sa compréhension patiente vis-à-vis de ce récit auquel l'auteur s'est efforcé de donner toutes les apparences du discours historique ! Encore une fois, l'exemple moral prévaut; la vraisemblance est encore une fois sacrifiée au critère de l'utilité qui oblitère décidément en Grèce ancienne toute velléité de distinction entre différents genres narratifs, en particulier selon le critère de leur valeur de vérité.

\section{Pratiques rituelles}

L'enquête sur la coïncidence de notre conception du rite avec les catégories grecques anciennes semble jouir, au premier abord, de meilleures perspectives que la recherche sur la taxinomie du récit à partir de notre notion du mythe.

\subsection{La notion de fête}

Les Grecs possèdent en effet dès Homère un lexème correspondant sinon au concept moderne de 'rite', en tout cas à celui de 'fête',

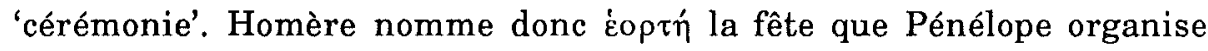
pour départager les prétendants, une célébration centrée sur le célèbre concours ( $\alpha_{\varepsilon} \theta \lambda_{\circ} \varsigma$ ) de tir à l'arc et dûment précédée d'une hécatombe en l'honneur d'Apollon ${ }^{37}$. Chez Alcman, c'est probablement Artémis qui est la protagoniste principale d'une éoprí destinée aux dieux; et quelques années plus tard, on voit Anacréon participer couronné à une "fête" célébrée en l'honneur de Dionysos. Quant à Pindare, il reconnaît dans

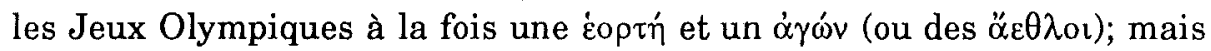

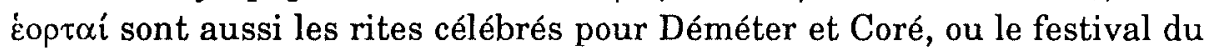
Delphinia consacré à Artémis et Apollon sur l'île d'Égine ${ }^{38}$.

À travers éop $\tau$ et et la permanence de son signifié, les Grecs semblent donc posséder un concept stable de la fête comprise comme 'célébration en l'honneur d'un dieu'. Il en va ainsi jusqu'à Hérodote et Aristophane, en attendant que Thucydide, une fois encore, vienne consacrer pour nous un changement important. Chez Hérodote en effet, autant les Héraia d'Argos que les Apatouries et les Brauronia athéniennes ou la

37 Hom., Od., XX, 156 et XXI, 258 (вор $\dot{\varepsilon} \kappa \alpha \tau \delta ́ \mu \beta \eta: X X, 276$.

38 Alcman, fr. 56, 2 Page, avec le commentaire proposé dans l'op. cit. (n. 19), p. 520 sq.; ANACR., fr. 410 Page; PIND., Ol., III, 34 sq.; V, 5 et VI, 69; Ném., XI, 27, etc.; Ol., VI, 95 et Pyth., VIII, 66. 


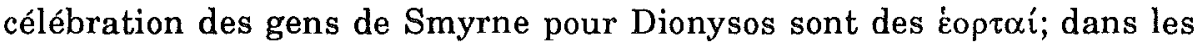
coutumes des peuples étrangers également, Hérodote reconnaît des "fêtes", tels les festivals que les Égyptiens organisent pour BastetArtémis ou Isis-Déméter. Pour Aristophane, ce sont les fameuses Anthestéries athéniennes qui représentent une żop cation du terme que viennent confirmer plusieurs inscriptions ${ }^{39}$. Thucydide en revanche, tout en employant ce même terme dans son sens traditionnel, lui attribue à l'occasion le signifié dérivé de 'temps

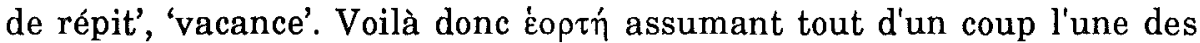
connotations préférées de son équivalent contemporain; il devient ainsi

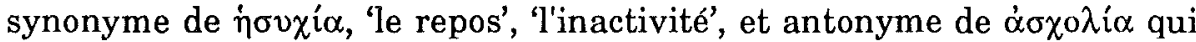
désigne l'absence de loisir qu'implique le labeur ${ }^{40}$. C'est d'ailleurs à la même époque que Démocrite, le fondateur de l'atomisme, déclarait qu'«une vie sans fête ( $\dot{\alpha} v \varepsilon o ́ \rho \tau \alpha \sigma \tau o \zeta)$ est comme un chemin sans auberge" ! Platon se situe dans une perspective analogue : célébrée en l'honneur des dieux, la fête est un don de ces mêmes dieux pour introduire une pause au sein de leurs labeurs ${ }^{41}$.

Cette conception de la fête comme loisir, comme un moment de repos interrompant le déroulement des occupations quotidiennes va naturellement être développée dans les textes chrétiens; on y assiste - fait caractéristique de l'extension d'un nouveau signifié - à la création de toute une série de néologismes formés à partir de $\dot{\varepsilon} o \rho \tau^{42}$. Ce terme continue bien entendu à désigner un ensemble de pratiques rituelles dédiées à la divinité, mais à cette désignation de la célébration s'ajoute une valeur de suspension régulière et temporaire de l'activité productrice; le caractère impératif de cette interruption est renforcé par les

39 Hdt., I, 31, 2; I, 147, 2 (cf. aussi Platon, Timée, 21b); VI, 138, 1; I, 150, 1, etc.;

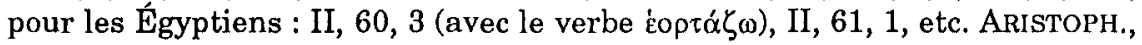
Acharn., 1079. Pour les inscriptions, voir les références données par J.D. Mikalson, The Heorte of Heortology, in GRBS, 23 (1982), p. 213-221, qui a dressé la liste de tous les rituels athéniens désignés comme éopraí (p. 220 n. 34).

40 THUC., II, 15, 2 (fête athénienne de synécisme, en l'honneur d'Athéna) et I, 70, 8;

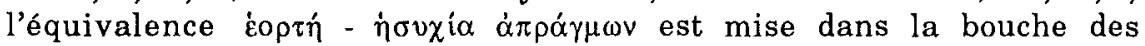
Corinthiens qui vantent chez les Athéniens, par contraste, leur constante activité : cf. L. EDMunds, Cleon, Knights, and Aristophanes' Politics, New York - London, 1987, p. 18 sq.

41 DÉmocR., fr. 68 B 230 Diels-Kranz; cf. également ANTIPHoN, fr. 87 B 57 DielsKranz. Platon, Lois, 653d, voir aussi Phèdre, 276b.

42 Voir notamment Luc, II, 41 et MatThIEU, XXVI, 5 (Pâques); Deutéronome, XVI, 16; LUC, XXII, 1 (la fềte des pains sans levain), etc.; mais on verra aussi

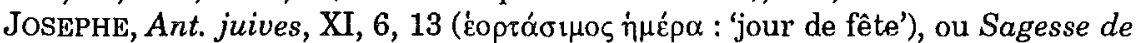

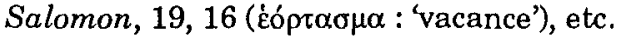


implications de la conception judéo-chrétienne du dimanche, jour du Seigneur. C'est par cet intermédiaire que l'on parvient à la conception de l'anthropologie culturelle et sociale contemporaine qui voit dans la fête et le rite une coupure avec le temps profane pour permettre l'insertion de leurs acteurs sociaux dans le temps primordial du sacré. Mais par l'emploi d'un concept qui trouve son fondement dans le classicisme grec et qui est réinterprété dans le cadre de la culture judéochrétienne, on court une fois encore le risque de plaquer sur la réalité ethnographique une catégorie assurément emblématique, mais non pertinente ${ }^{43}$. Le processus de projection qui, avec le mythe, se réalisait au niveau du signifiant, se produit ici sur le plan du signifié.

\subsection{Désignations par synecdoque}

En deçà de la supposée rupture festive, il convient donc de revenir aux catégories proprement grecques, à leurs signifiants et à leur manière de découper le champ du rite et de la cérémonie. On trouve à côté du lexème central de غ́op synonymes. Si l'on reprend le texte d'Hérodote sur les rites et les cultes des Égyptiens, on rencontre d'abord $\pi \alpha v \eta \dot{\gamma} \gamma \rho\llcorner\varsigma$ (et $\pi \alpha v \eta \gamma v \rho i \zeta \omega$ ), un terme qu'utilisent peut-être Archiloque (fête de Déméter et Coré) et certainement Pindare (festival de Zeus Lycaios et Jeux panhelléniques) comme aussi Thucydide (cérémonies accomplies par les Corcyréens). Quant à Hérodote lui-même, il ne limite pas l'emploi de ce terme à la désignation des festivités égyptiennes, mais il l'utilise, avec غ́oprń, pour dénoter les Héraia d'Argos où Cléobis et Biton trouvent la mort et, de manière plus générale, les célébrations quadriennales des Athéniens ${ }^{44}$. Conformément à son étymologie, $\pi \alpha v \eta ́ \gamma v \rho ı \varsigma$ implique une idée de rassemblement et c'est bien là son signifié générique : la réunion à laquelle renvoie ce terme n'a pas forcément de rapport avec un culte rendu à un dieu. C'est donc le rassemblement dont la fête est l'occasion qui a conduit ce terme à désigner le rite dans son ensemble.

43 Voir notamment E. LEACH, Critique de l'anthropologie, Paris, 1968 (éd. or. : London, 19662), p. 223 sq., et M. ELIADE, Le sacré et le profane, Paris, 1965 (éd. or. : Hamburg, 1957), p. 74 sq.; j'ai présenté à ce propos quelques remarques critiques dans Morfologia e funzione della festa nell'Antichità, in AION, 4/5 (1982/3), p. 3-23. La distinction entre "sacré» et "profane" remonte - rappelonsle - à É. DuRKheIm, Les formes élémentaires de la vie religieuse. Le système totémique en Australie, Paris, $1915^{2}$, p. 50 sq.

44 HDT., II, 58 sq.; ARCHIL., fr. 322 West; PIND., Ol., IX, 96 et Isthm., IV, 28; THUC., I, 25,$4 ;$ HDT., I, 31, 2 sq. et VI, 111, 2. I faut ajouter le terme épotı que l'on trouve chez EUR., Electre, 625 : cf. ChaNTraIne, op. cit. (n. 8), p. 374. 
Ce recours à l'un des caractères distinctifs du rite pour en désigner l'ensemble, par un processus de synecdoque de la partie pour le tout, est en fait une constante dans le système grec de la désignation des occasions rituelles. Il en va de même pour le lexème $\pi \alpha v \vee v \chi i \varsigma$ $(\pi \alpha v v v \chi i \zeta \omega)$ qui renvoie à toute activité s'étendant sur la nuit entière, notamment une cérémonie rituelle et religieuse: célébration pour Déméter, mystères d'Éleusis ou festival en l'honneur de Méter ${ }^{45}$. Ce procédé de désignation par synecdoque est sensible dès les textes homériques puisque, comme cela a été mentionné, la grande fête des prétendants placée sous l'égide d'Apollon mérite aussi bien la désignation de

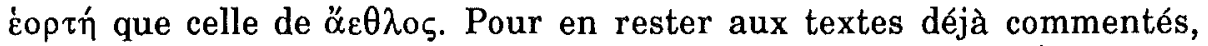
Hérodote, dans son catalogue des célébrations rituelles des Égyptiens, cite à côté des $\pi \alpha v \eta \gamma u ́ p \iota \alpha$, les $\pi$ o $\mu \pi \alpha i$, 'les processions', et les $\pi \rho \circ \sigma \alpha \gamma \omega \gamma \alpha i$, un terme rarement employé dans ce contexte festif et qui désigne probablement l'introduction à la cérémonie. Platon, quant à lui, dans un passage du Banquet, fait des actes constitutifs de la fête - les danses

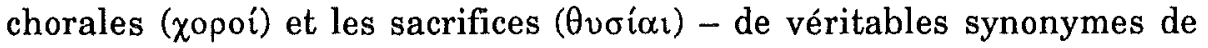

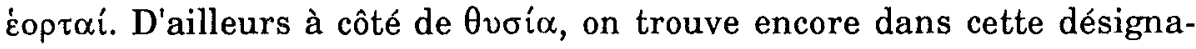

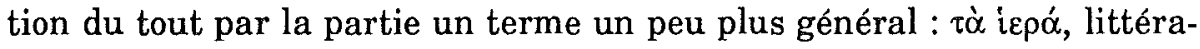
lement 'les actions sacrées'; d'Homère à Hérodote, on désigne de ce lexème aussi bien le sacrifice que les actes rituels qui en constituent le contexte ${ }^{46}$.

Le rite, c'est donc en Grèce la fête ou l'action qui en forme le pivot. Comme on a tenté de le montrer ailleurs, les Grecs aimaient à désigner la célébration rituelle du nom de l'action située en son centre : le

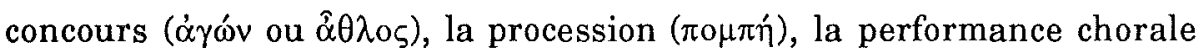

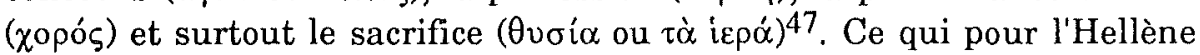

45 Eur., Hélène, 1365; Aristoph., Gren., 371 et 446; HDT., IV, 76, 3; voir déjà SAPPHO, fr. 23, 13 et fr. 30, 3 Voigt.

46 Platon, Banquet, 197d; Hom., Il., I, 147 (sacrifice à Apollon), XXXIII, 195 et 209 (sacrifice à Borée et Zéphyr); HDT., II, 63, 1 (rites de Paprémis) et IV, 33, 5 (sacrifice et rite thraces pour Artémis, cf. aussi V, 67, 4). Voir encore HIPPOCR., Airs, XVII, 3. Les variations relevées par J. CASABONA, Recherches sur le vocabulaire des sacrifices en Grèce, des origines à la fin de l'époque classique, Paris, 1966, p. 132 sq., dans l'emploi de $\theta v \sigma i \alpha$, qui désigne tour à tour le tout et la partie, dépendent de ce phénomène de désignation par synecdoque. Sur la signification de $\tau \grave{\alpha}$ ípó, on lira J. RudHARDT, Notions fondamentales de la pensée religieuse et actes constitutifs du culte dans la Grèce classique, Genève, 1958, p. 23 sq., et CASABONA, op. cit., p. 5 sq.

47 Voir l'art. cit. (n. 43), ainsi que W. BURKERT, Griechische Religion der archaischen und klassischen Epoche, Stuttgart - Berlin, 1977, p. 163 sq. 
est donc essentiel dans ce que nous concevons comme un rite, c'est autant la relation à une divinité que le type d'acte qui y est accompli. L'accent mis sur l'action va même jusqu'à influencer la dénomination

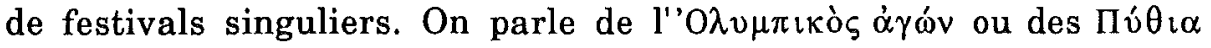
$\dot{\alpha} \varepsilon \hat{\theta} \lambda \alpha$, 'joutes olympiques' ou 'combats pythiques' : par ces désignations l'attention est focalisée sur le concours qui est au centre du rituel. Mais il y aussi les Thesmophories, les Oschophories ou la Daphnéphorie où la dénomination singulière $d u$ rituel concerné fait explicitement référence aux objets portés lors de la procession qui en constitue le cœur.

À ces nombreuses désignations de la célébration religieuse par synecdoque, il faut encore ajouter celles qui font référence non seulement à un acte constitutif particulier, mais aussi à l'atmosphère qui l'entoure. Ainsi en va-t-il de $\pi \alpha i \zeta \varepsilon$ เv et de ses dérivés qui, dans le contexte de la fête, représentent une allusion au divertissement et à la réjouissance que constituent les danses chorales, les luttes gymniques ou les concours musicaux ${ }^{48}$. Inversement à la procédure de la synecdoque, la pratique rituelle peut aussi être désignée en Grèce par un terme au signifié plus large. Il en va ainsi de l'emploi de $\tau \grave{\alpha}$ vo ou $\tau \grave{\alpha}$ vó $\mu \imath \mu \alpha$ qui dénotent en général les pratiques sanctionnées par la coutume, et en particulier les rites religieux. Quand ces termes sont employés dans ce sens spécifique, l'adjonction d'une qualification en délimite volontiers le champ d'application; ainsi, en utilisant ce terme,

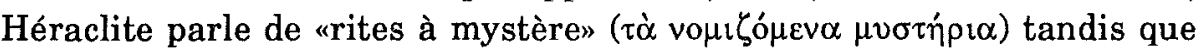

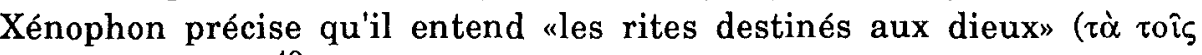

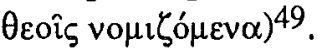

\subsection{Les cultes à mystère}

Mais dans ce rapide balayage du champ lexical et du champ sémantique du rituel grec, on n'a pas encore rencontré le $\delta \rho \omega ́ \mu \varepsilon v o v$ cher à Jane Harrison! C'est que le verbe $\delta \rho \alpha \dot{\omega} \omega$ et l'acte en train de s'accomplir que

L'analyse des textes épigraphiques entreprise par MIKALSON, art. cit. (n. 39), p. 217 sq., conduit à des résultats convergents.

48 Cf. ARISTOPH., Gren., 384 sq. et 816; PLATON, Lois, 657d; ARISTOTE, Rhét., 1380b 3, etc.; voir à ce propos MiKALSON, art. cit. (n. 39), p. 215 sq., et CASABONA, op. cit. (n. 46), p. 131 sq.

49 HÉraCl., fr. 22 B 14 Diels-Kranz; XÉNOPHON, Cyr., IV, 5, 14 ainsi que I.G., I², 22, 4; ANTIPHON, V, 82; EUR., Bacch., 70 sq. ou Suppl., 19. On ajoutera l'expression

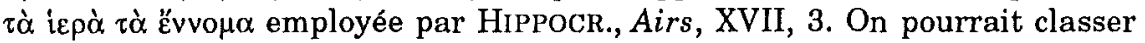

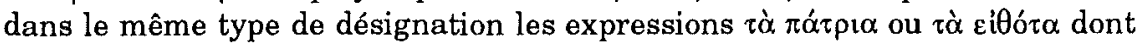
les utilisations dans ce sens sont données par J. D. Mikalson, Athenian Popular Religion, Chapel Hill - London, 1983, p. 96 sq. 
dénote sa forme participiale $\delta \rho \omega ́ \mu \varepsilon v o v$ interviennent tardivement dans la série des lexèmes au service de la désignation de la célébration rituelle. Si à l'époque classique le verbe $\delta \rho \alpha \hat{v}$ est probablement employé pour dénoter l'action cultuelle dans quelques inscriptions, en revanche dans les textes littéraires il sert essentiellement la désignation de l'action dramatique. Dans cette acception de l'agir scénique et en

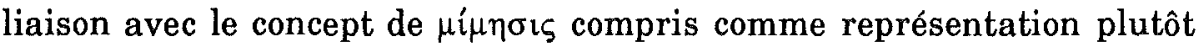
qu'imitation, il est un élément constitutif central dans la définition aristotélicienne de la tragédie et de la comédie ${ }^{50}$. Ce n'est guère qu'au début de notre ère, avec Plutarque puis Pausanias, que le $\delta \rho \omega ́ \mu \varepsilon v o v$ fait son entrée dans le champ lexical de ce que nous considérons constituer un rite, mais avec cette limitation déterminante que $\delta \rho \hat{\alpha} v$ et $\delta \rho \omega ́ \mu \varepsilon v o v$ désignent exclusivement les actes des rites à mystère. Plutarque use en effet de ces termes dans la description des mystères destinés à Déméter et dans celle des rites dionysiaques. Et de même Pausanias limite-t-il l'usage de $\delta \rho \omega ́ \mu \varepsilon v o v$ au contexte des rites à mystère, du type de ceux célébrés en l'honneur de Déméter ou de Dionysos ${ }^{51}$.

Cette incursion dans la désignation des rites à mystère nous fait aborder du même coup un nouveau champ lexical qui inclut des termes plus techniques, en correspondance avec la spécificité de son domaine

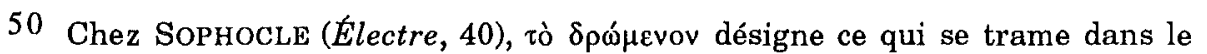
palais de Clytemnestre (cf. aussi $E$ dipe à Colone, 1644), et chez THUCYDIDE (V, 102), il dénote l'acte de guerre. On le trouve dans un sens religieux mais à titre de conjecture dans $I . G ., I^{2}, 4$, 4; voir aussi 188, 55 (verbe $\delta \rho \hat{v}$ ). Il est peut-être à l'origine du terme $\delta \rho \eta \sigma \mu o \sigma u ́ v \eta$ que PaUSANIAS (II, 14, 3) restitue dans l'Hymne homérique à Déméter, 476 : cf. N.J. RICHARDSON, The Homeric Hymn to Demeter, Oxford, 1974, p. 303 sq., avec une étude morphologique de ce verbe. Pour une analyse sémantique, voir H. Schreckenberg, DRAMA. Vom Werden der griechischen Tragödie aus dem Tanz, Würzburg, 1960, p. 1 sq. et p. 49 sq. Pour le sens à proprement parler dramatique de $\delta \rho \hat{\alpha} v$, voir ARISTOTE, Poét., 1448a 24 sq., avec le commentaire de R. DuPONT-Roc et J. LALlor, Aristote, La Poétique, Paris, 1980, p. 160 sq.

51 Emplois des formes de $\delta \rho \hat{\alpha} v$ chez Plutarque : Mor., 352c, 378ab, 293d et fr.

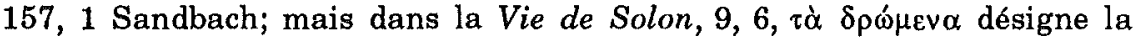
cérémonie rappelant la victoire que les Athéniens remportèrent sur les Mégariens à l'époque du législateur. Emplois chez PAUSANIAS : I, 43, 2 (rite lié au culte de Déméter et Coré à Mégare), II, 22, 3 (idem à Argos), II, 37, 2 (idem à Lerne; aussi $\tau \varepsilon \lambda \varepsilon \tau$ ), III, 20, 5 (idem dans le Taygète), II, 37, 6 (rites secrets pour Dionysos à Lerne), III, 22, 2 (culte de Dionysos près de Gythion; dit aussi غ́oprí), XI, 27, 2 et 30, 12 (culte probablement orphique d'Éros), II, 14, 1, V, 10, 1, VIII, 15, 1 et 31, 7 ainsi que $X, 31,11$ (mystères d'Éleusis); voir aussi ATHÉNÉE, XIV, 660a. On lira à ce sujet l'analyse de SchrECKENBERG, op. cit. (n. 50), p. $122 \mathrm{sq}$. 
de référence, celui des cultes initiatiques. En effet, en plus des dérivés de $\delta \rho \alpha \hat{v}$, les rites à mystère sont souvent désignés par le lexème $\tau \varepsilon \lambda \varepsilon \tau \eta ́$, un terme qu'on a laissé pour compte jusqu'ici comme a été consciemment omis celui de $\mu v \sigma \tau \eta ́ p \imath \alpha$. C'est que $\tau \varepsilon \lambda \varepsilon \tau \eta ́$ renvoie de manière spécifique aux actes rituels des cultes initiatiques et mystiques, selon la définition très précise proposée par Athénée quand il dit : «Nous appelons $\tau \varepsilon \lambda \varepsilon \tau \alpha i$ les fêtes ( $\varepsilon \rho \rho \tau \alpha \hat{i})$ qui dépendent de la tradition mystique». Ce lexème ne concerne donc qu'une catégorie spécifique de festivités, celle des rites d'initiation marqués du sceau du secret: les Grecs incluaient dans ce qui apparait ainsi comme une catégorie aux contours plus précis les Thesmophories, les rites dionysiaques, le culte rendu aux Corybantes et les rites orphiques. Mais à la fin de l'époque archaïque, notamment chez Pindare, $\tau \varepsilon \lambda \varepsilon \tau \eta \dot{~ a s s u m e ~ e n c o r e ~ u n ~ s i g n i f i e ́ ~}$ moins spécifique, voisin de celui du concept plus général impliqué par

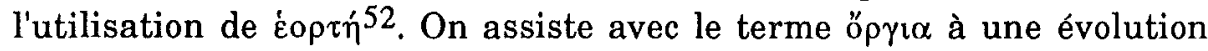
sémantique parallèle puisque ce terme perd rapidement son sens général $\mathrm{d}^{\prime \prime a c t i o n ~ c u l t u e l l e ' ~ p o u r ~ s e ~ s p e ́ c i a l i s e r ~ d a n s ~ l a ~ d e ́ s i g n a t i o n ~ d e s ~}$ pratiques initiatiques marquées par le secret rituel ${ }^{53}$. Quant à la dénomination $\mu v \sigma \tau \eta \dot{\rho} \rho \iota$, dont dérive le verbe $\mu v \varepsilon i ̂ v$ 'initier', si elle s'est développée dans l'ambiance athénienne au lieu d'avoir l'origine

52 ATHÉNÉE, II, 40d. Les rites suivants sont désignés en Grèce classique par le terme $\tau \varepsilon \lambda \varepsilon \tau \dot{\eta}$ : les Thesmophories chez HDT., II, 171, 2; les rites du culte de

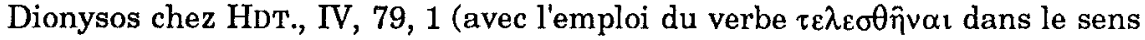
de 'être initié'), ARISTOPH., Gren., 342 et 368, ou Eur., Bacch., 22 et 73; le culte des Corybantes chez Aristoph., Guêpes, 121 et Platon, Euthyd., 277d; les mystères orphiques chez ARISTOPH., Gren., 1032; Platon, Rép., 365a et Protag., $316 \mathrm{~d}$. Le verbe $\tau \varepsilon \lambda \hat{\varepsilon} \omega$ est aussi employé par Hérodợ dans la désignation des mystères (ő $\rho \imath \alpha$ ) : voir par exemple II, 51, 2 (mystères des Cabires). - On trouvera $\tau \varepsilon \lambda \varepsilon \tau \dot{\eta}$ employé dans un sens plus large chez PIND., Ol., III, 41 (cérémonies pour les dieux), Pyth., IX, 97 (festival de Pallas à Cyrène) et Ném., $\mathrm{X}, 34$ (les Panathénées); il en va de même chez Aristoph., Paix, 413 et 419 , où ce terme recouvre à la fois les Panathénées, les Mystères (d'Éleusis), les Dipolies et les Adonies : voir à ce sujet A. BRELICH, Gli eroi greci. Un problema storico-religioso, Roma, 1958, p. 199 sq., et les études citées par W. BURKERT, Ancient Mystery Cults, Cambridge Mass. - London, 1987, p. 137 n. 40 et 41.

53 Chez EsChyle (Sept, 172 sq.), öpyı est encore synonyme de ípó (mais cf. fr. 57 Radt), et chez SOPHOCLE (Trach., 765 et Antig., 1013), ce terme se réfère à un acte de sacrifice. Mais dans l'Hymne homérique à Déméter, 173 et 476 , il a déjà un sens initiatique : cf. RICHARDSON, op. cit. (n. 50), p. 251 et 304 sq., et A. Henrichs, Die Maenaden von Milet, in ZPE, 4 (1969), p. 223-241, qui cite d'autres attestations de cet emploi particulier de ǒp $\gamma$ io. Cf. encore PLATON, Lois, $717 \mathrm{~b}$, avec le commentaire de L. ZIEHEN, art. Orgia, in RE, XVIII, 1 (1939), col. 1026-1029. 
mycénienne qu'on a voulu lui attribuer, elle pourrait être le lieu d'un double synecdoque : $\mu \nu \varepsilon \hat{v} v$ pourrait se référer dans un premier temps à l'une des phases des Mystères d'Éleusis, avant que $\mu \nu \sigma \tau \eta \dot{\rho} \iota \alpha$ de la désignation de l'ensemble de la célébration ne passe à celle de toute cérémonie initiatique ${ }^{54}$.

Preuves supplémentaires, s'il en faut, de la multiplicité des lexèmes susceptibles de recouvrir partiellement le concept moderne de rite ! Le savoir issu des découpages et des distinctions hiérarchiques que ces nomenclatures opèrent dans le champ sémantique des manifestations cérémonielles est délibérément placé sous le signe de la complexité et de l'absence d'homogénéité. Sans parler de la distinction qui commence à se dessiner à partir de Démocrite et Thucydide entre un temps de labeur et des moments de loisir coïncidant avec des célébrations rituelles et sans analyser davantage les relations d'inclusion hiérarchique organisant les signifiés des termes découpant le champ sémantique du rite, on peut affirmer que l'équivalent grec du concept forgé par l'anthropologie culturelle et sociale possède en fait les limites floues que lui assignent les multiples occasions et formes de célébration rituelle et cultuelle.

\section{Nomenclatures sans système}

Même rapide, l'enquête auprès de nos informateurs lettrés n'est parvenue à déceler ni signifiants, ni signifiés correspondant exactement dans la culture indigène aux domaines que nous entendons délimiter par les termes de mythe et de rite. Dans ces deux cas, le mode de désignation grec est essentiellement tributaire de l'occasion et de la réception du récit ou de l'acte cultuel concernés; en raison de la multiplicité des lexèmes dès lors impliqués l'enquête devrait se poursuivre dans la direction de la dénomination des genres poétiques d'un côté et dans celle des différents festivals de l'autre. Mais revenons aux deux termes susceptibles de coiffer chacun des domaines que semblent circonscrire les catégories modernes. Au moment où l'on pense déceler

54 Sur l'emploi de $\mu v \varepsilon i ̂ v$ et de ses parents pour désigner un grade initiatique dans les Mystères d'Éleusis, cf. I.G., I², 6B, 49 sq. et PluT., Alcib., 22, 3, avec le commentaire de Richardson, op. cit. (n. 50), p. 20 sq. Chez HÉrodoTE, ce verbe correspond aussi bien à l'initiation aux Mystères d'Éleusis (VIII, 65, 4 : une

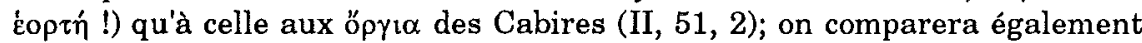
ARISTOPH., Gren., 887 avec 370 et 456 . Sur le problème de l'éventuelle origine mycénienne de la racine $\mu v(\sigma)$ - voir BURKERT, op. cit. (n. 52), p. 8 sq., et sur celui de sa signification première, CHANTRAINE, op. cit. (n. 8), p. 728 sq. 
dans le signifié de $\mu \hat{v} \theta$ o désigné, la vraisemblance se définit non pas du point de vue factuel, mais selon un critère éthique. Et quand on croit saisir à travers le terme

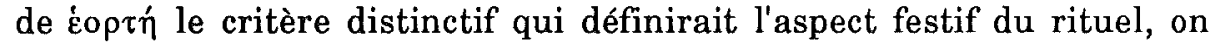
s'aperçoit que les célébrations subsumées sous ce terme peuvent tout aussi bien être désignées, suivant leurs circonstances d'exécution, par l'acte cultuel qui en constitue le pôle.

C'est dire qu'en Grèce ancienne aussi bien que dans l'anthropologie moderne, il n'y a de définition qu'opératoire de ces manifestations symboliques que sont devenus à nos yeux mythes et rites. Pour un Hérodote comme pour nous, ces catégories ne peuvent être que relativisées par la confrontation avec celles d'autres cultures. Par ailleurs, classant des produits langagiers ou gestuels du processus symbolique, elles ne peuvent plus se contenter d'être l'objet de notre part d'une simple analyse lexicale et sémantique de type structural. Leur étude requiert l'interrogation des circonstances de leur formulation et de leur énonciation, avec les implications pratiques de toute mise en discours.

Université de Lausanne

Claude CALAME

Faculté des Lettres

Section des Sciences de l'Antiquité

BFSH 2, 4026

$\mathrm{CH}$ - 1015 LAUSANNE 